\title{
Experimentelle Untersuchung über die schädliche Wirkung von ungesättigten Fettsäuren. (Vorläufige Mitteilung).
}

\author{
Von \\ Shuzo Yonechi. \\ (米地秀三) \\ (Aus Prof. Koyanagis Augenklinik der Tohoku- \\ Universität in Sendai.)
}

Es ist bereits wiederholt gesagt worden, dass bei chronischen Nierenleiden mit Retinitis nephritica das Blut oft beträchtlich reich an Cholesterin ist, wie gewisse Autoren derzeit sehr geneigt sind, im hohen Cholesteringehalt des Blutes hier die eigentliche Ursache der nephritischen Netzhautveränderungen zu erblicken.

Bei der Beurteilung der Hypercholesterinämie muss man aber doch zugleich darauf Rücksicht nehmen, dass im Tierkörper, nicht nur normalerweise, sondern auch unter pathologischen Umständen das Cholesterin und die Phosphatide immer nebeneinander, und zwar in einem ziemlich konstanten Verhältnis vorkommen, welches sich heute als den lipozytischen Koeffizienten nach Ma yer und Schaeffer bezeichnet. Während das Cholesteriuproblem bei Retinitis nephritica schon vielfach der Gegenstand von Forschungen über ihre Pathogenese war, kommt es bisher fast gar nicht in Betracht, ob eine Prävalenz der Phosphatide, wenn es dabei der Fall ist, eine gewisse Schädlichkeit auf den Organismus auszuüben vermag.

Vor kurzem fand Litzner ${ }^{12}$ zufälligerweise, dass sich bei Schrumpfniere die basophilen Erythrozyten im peripheren Blut vermehren. Bald nachher konnte auch Koyanagi, ${ }^{2}$ freilich von der Angabe Litzners völlig unbeeinflusst, sondern von einem recht rationellen Gedanken ausgehend, feststellen, dass bei Retinitis nephritica eine bedeutende Vermehrung der basophilen Tüpfelzellen des Blutes für ein diagnostisches Merkmal gilt. Nach der sorgfältigen Auseinan-

1) Litzner, Med. Klinik $1933,81$.

2) Kovanagi, Klin. M. f. Augenh. 1933, 91, 168. 
dersetzung gab Koy an ag $\mathrm{i}^{2)}$ mit einer gewissen Wahrscheinlichkeit an, dass es sich in der genannten Blutveränderung bei Retinitis nephritica wesentlich um eine schädliche Wirkung der Phosphatide mit intravitale. Hämolyse handelt, wie sie beim Kaninchen mit Lezithinfütterung bereits von $\mathrm{Brink}$ man und $\mathrm{Dam}^{3)}$ experimentell bewiesen wurde. Koyanagi zog dabei für das bessere Verständnis über die Wirkungsweise der Phosphatide als Beispiel die Toluylendiaminvergiftung heran, welche hier vielleicht auf einen erhöhten Gehalt an ungesättigten Fettsäuren im Blute zurückzuführen ist.

Unter dieser Auffassung ist es sehr erwünscht, zu wissen, ob und wie weit die genannten Substanzen beim Tierversuche pathologische Erscheinungen hervorrufen, die gerade auf die Beurteilung der noch immer strittigen Pathogenese der Retinitis nephritica gewisses Licht werfen würden.

I. Blutveränderungen bei Kaninchen mit Toluylendiaminvergiftung.

Das Toluylendiamin ist bekanntlich ein stark hämolytisches Gift, dessen ganz kleine Dose hier als zweckmässig anwendbar ist. Der betreffende Versuch, bei dem 4 ausgewachsene ca. $2 \mathrm{~kg}$ schwere Kaninchen zu Gebote standen, erstreckte sich auf eine Zeitdauer von 7 Wochen fortlaufend, nämlich von 10. Januar bis 28. Februar 1934. In den ersten 3 Wochen erhielten die sämtlichen Tiere jeden zweiten Tag. $3 \mathrm{ccm}$ einer $1 \%$ igen Toluylendiaminlösung subkutan, durch die weiteren 10 Tag aber täglich $5 \mathrm{ccm}$ derselben Konzentration und während der restlichen Versuchszeit auch täglich $5 \mathrm{ccm}$ seiner $2 \%$ igen Lösung.

Tabelle 1.

(Kaninchen Nr.6)

\begin{tabular}{l|r|r|r|r|r|r|r|r}
\hline Versuchszeit (Woche) & Anfang & 1. W. & 2. W. & 3. W. & 4. W. & 5. W. & 6. W. & 7. W. \\
\hline Gewicht (g) & 1935 & 1940 & 1920 & 1915 & 2130 & 2050 & 1850 & 1800 \\
Erythrozyten & 6,44 & 5,92 & 5,85 & 5,70 & 5,67 & 5,89 & 5,62 & 5,29 \\
(Mrillionl) & 8800 & 9200 & 12500 & 13900 & 12140 & 10750 & 11400 & 13400 \\
Lenkozyten & 15,24 & 14,27 & 13,03 & 11,41 & 12,32 & 12,73 & 12,58 & 12,52 \\
Hb-Gehalt & & & & & \\
(B ürker) & 5 & 17 & 11 & 21 & 16 & 7 & 4 & 5 \\
Basophile & & & & & \\
Punktierung & 68 & 106 & 112 & 234 & 222 & 210 & 186 & 192 \\
Polychromasie & & & & & &
\end{tabular}

3) Brinkman u. Dam, Bioch. Zeitschr. 1920, 108, 35. 
Die Blutuntersuchungen wurden bei jedem Versuchstier wöchentlich einmal ausgeführt, wobei ich das Blut immer in einer bestimmten Zeit nachmittags entnahm, um mögliche Beeinflussung von der vormittägigen Nahrungsaufnahme auszuschliessen. Von wesentlicls iibereinstimmenden Resultaten dieser Versuchsreihe wird hier nur ein Beispiel (Kaninchen Nr. 6) in der Tabelle 1 angegeben, um unnützliche Wiederholung. zu vermeiden. Und in Fig. 1 stellen sich zeitliche Schwankungen der einzelnen Blutbestandteile bei einem anderen Fall (Kaninchen Nr. 8) graphisch dar.

II. Blutveränderungen bei Kaninchen mit Sonderfütterung von ungesättigten Fettsäuren.

Der Versuch wurde hier zuerst unter Anwendung von dem Perillaöl angestellt, welches nach der Literaturangabe ungesättigte Fettsäuren in beträchtlichem Masse (ca. 80\%) enthält. Die Versuchszeit

Tabelle 2.

(Kaninchen Nr. 10)

\begin{tabular}{|c|c|c|c|c|c|c|c|c|}
\hline Versuchszeit (Woche) & Anfang & 1. W. & 2. W. & 3. W. & 4. W. & 5. W. & 6. W. & 7. W. \\
\hline $\mathrm{Ge}$ & 2355 & 2275 & 2245 & 2260 & 2295 & 2270 & 2210 & 2100 \\
\hline $\begin{array}{c}\text { Elythrozyten } \\
\text { (Million) }\end{array}$ & - & 30 & 6,38 & 5,98 & 6,10 & 6,15 & 4,91 & 4,77 \\
\hline Leukozyten & 6280 & 9430 & 14080 & 12780 & 13700 & 16300 & 13950 & 12500 \\
\hline $\begin{array}{l}\text { Hb-Gehalt } \\
\quad \text { (Bürker) }\end{array}$ & 18 & 15,27 & 15,36 & 14,54 & 14,49 & 14,39 & 12,41 & 11,84 \\
\hline $\begin{array}{l}\text { Basophile } \\
\text { Punktierung }\end{array}$ & 0 & 12 & 15 & 13 & $\tilde{0}$ & 9 & 4 & 0 \\
\hline Polychromasie & 66 & 168 & 85 & 120 & 125 & 120 & 158 & 238 \\
\hline
\end{tabular}


Fig. 2.

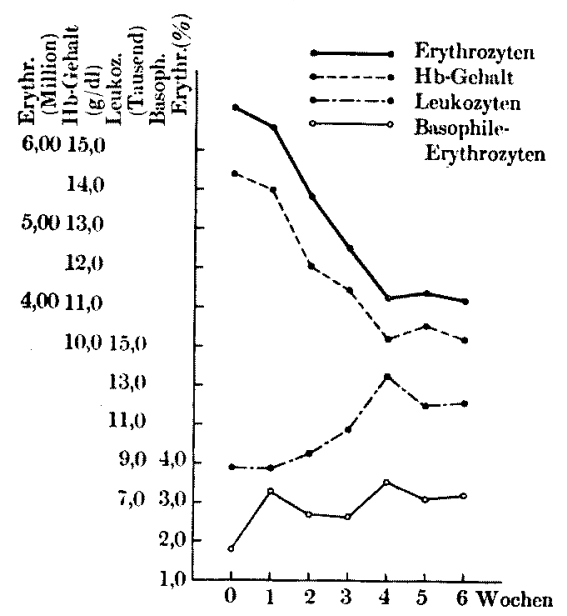

dauerte ebenfalls 7 Wochen lang fortlaufend, nämlich von 6. Januar bis 28. Februar 1934. Die Tiere wurden neben der gewöhnlichen Laboratoriumnahrung auch mit diesem Öl in der Weise gefüttert, dass die tägliche Dosis desselben während der ganzen Versuchszeit allmählich von $3 \mathrm{~g}$ bis auf $7 \mathrm{~g}$ gesteigert war, d.h. in den ersten 2 Wochen $3-4 \mathrm{~g}$, durch die weiteren 10 Tage 5-6 g und in den übrigen Versuchstagen $7 \mathrm{~g}$ betrug. Das Versuchsergebnis bei einem Tier

(Kaninchen Nr.10) veranschaulicht die Tabelle 2 beispielsweise.

In der zweiten Versuchsreihe benützte ich Leinölsäure, die ein Gemisch von ungefähr gleichen Teilen Linol- und Linolensäuren ist und als Handelspräparat von der Firma Theodor Schuchardt hergestellt wurde. In jedem Versuchstier wurde eine bestimmte Dosis derselben per os gegeben, und zwar in den ersten 2 Wochen täglich $3 \mathrm{~g}$ und durch die weiteren 4 Wochen 5-6 g. Ein Beispiel dieser Versuchsreihe, die wesentlich miteinander übereinstimmende Blutveränderungen aufwies, ist in der Tabelle 3 wiedergegeben. Und Fig. 2 macht das Resultat bei einen anderen Fall (Kaninchen Nr. 16) leicht ersichtlich.

\section{Tabelle 3.}

(Kaninchen Nr. 14)

\begin{tabular}{l|r|r|r|r|r|r|r}
\hline Versuchszeit(Woche) & Anfang & 1. W. & 2. W. & 3. W. & 4. W. & 5. W. & 6. W. \\
\cline { 1 - 8 } & 1835 & 1750 & 1800 & 1820 & 1800 & 1750 & 1750 \\
Gerwicht (g) & 6,27 & 5,58 & 5,65 & 5,85 & 5,64 & 5,41 & 5,11 \\
$\begin{array}{c}\text { Erythrozyten } \\
\text { (Hillion) }\end{array}$ & 7160 & 6720 & 8050 & 8250 & 13700 & 9850 & 8600 \\
Lenkozyten & 12,94 & 11,71 & 12,02 & 13,37 & 12,28 & 12,16 & 12,01 \\
$\begin{array}{c}\text { Hb-Gehalt } \\
\text { (B ïrker) }\end{array}$ & 10 & 10 & 15 & 14 & 13 & 13 & 6 \\
$\begin{array}{c}\text { Basophile } \\
\text { Punktierung }\end{array}$ & 81 & 114 & 192 & 154 & 142 & 130 & 126 \\
Polychromasie & & & &
\end{tabular}


III. Wasserwechselstörungen bei Kaninchen mit Toluylendiaminvergiftung.

A. Intermediärer Wasseraustausch. Bei jedem Versuchstier wurde $150 \mathrm{ccm}$ lauwarme physiologische Kochsalzlösung mit Zusatz von 1,5-2 $\mathrm{g}$ des Toluylendiamins peroral dargereicht. Darauffolgend wurde die Hb-Bestimmung als Indikator für den Wassergehalt des Blutes halbstündlich, und zwar im Verlauf von 5 Stunden wiederholt vorgenommen, wie die Tabelle 4 das Resultat veranschaulicht.

\section{Tabelle 4.}

Zeitliche Schwaukungen des Hb-Gehaltes (Bürker) nach der Wasseraufnahme.

\begin{tabular}{c|c|c|c|c|c|c|c|c|c|c|c}
\hline $\begin{array}{c}\text { Nr.d. d. Gewicht } \\
\text { Tiere }\end{array}$ & $\begin{array}{c}\text { Anfang } \\
\text { (g) }\end{array}$ & $\frac{1}{2}$ St. & 1 St. & $1 \frac{1}{2}$ St. & 2 St. & $2 \frac{1}{2}$ St. & 3 St. & $3 \frac{1}{2}$ St. & 4 St. & $4 \frac{1}{2}$ St. \\
\hline 17 & 1955 & 17,63 & 17,55 & 15,20 & 14,79 & 15,87 & 17,43 & 16,70 & 17,51 & 16,81 & 18,04 \\
18 & 2050 & 16,63 & 15,05 & 14,76 & 14,29 & 15,11 & 15,08 & 15,74 & 14,64 & 16,49 & 16,14 \\
19 & 1935 & 15,27 & 14,91 & 13,85 & 13,39 & 14,00 & 13,75 & 13,14 & 13,51 & 13,98 & 12,68 \\
20 & 1935 & 15,23 & 13,93 & 14,00 & 15,67 & 15,87 & 15,48 & 16,14 & 16,59 & 16,52 & 14,91 \\
21 & 1980 & 16,31 & 14,94 & 14,40 & 13,78 & 14,27 & 15,74 & 15,02 & 15,42 & 15,36 & 16,67
\end{tabular}

B. Renale Wasserausscheidung. Auch hier wurde die perorale Wasserdarreichung mit gleichzeitiger Toluylendiaminvergiftung bei jedem Tier unter den völlig gleichen Bedingungen ausgeführt, wie im soeben beschriebenen Versuche. Das Resultat macht sich in der Tabelle 5 übersichtlich.

$$
\text { Tabelle } 5 \text {. }
$$

Halbstündliche Bestimmungen der Harnmenge nach der Wasseraufnahme (ccm).

\begin{tabular}{r|c|c|c|c|r|r|r|r|r|r|r|r|r|r|r}
\hline $\begin{array}{l}\text { Nr.d. } \\
\text { Tiere }\end{array}$ & $\begin{array}{c}\text { Gewicht } \\
\text { (g) }\end{array}$ & $\begin{array}{c}\frac{1}{2} \\
\text { St. }\end{array}$ & $\begin{array}{c}1 \\
\text { St. }\end{array}$ & $\begin{array}{c}1 \frac{1}{2} \\
\text { St. }\end{array}$ & $\begin{array}{c}2 \\
\text { St. }\end{array}$ & $\begin{array}{c}2 \frac{1}{2} \\
\text { St. }\end{array}$ & $\begin{array}{c}3 \\
\text { St. }\end{array}$ & $\begin{array}{c}3 \frac{1}{2} \\
\text { St. }\end{array}$ & $\begin{array}{c}4 \\
\text { St. }\end{array}$ & $\begin{array}{c}4 \frac{1}{2} \\
\text { St. }\end{array}$ & $\begin{array}{c}5 \\
\text { St. }\end{array}$ & $\begin{array}{c}5 \frac{1}{2} \\
\text { St. }\end{array}$ & $\begin{array}{c}6 \\
\text { St. }\end{array}$ & $\begin{array}{c}6 \frac{1}{2} \\
\text { St. }\end{array}$ & $\begin{array}{c}7 \\
\text { St. }\end{array}$ \\
\hline 22 & 2125 & 3 & 0 & 0 & 0 & 3 & 5 & 15 & 20 & 35 & 12 & 25 & 23 & 15 & - \\
23 & 2210 & 0 & 0 & 10 & 20 & 18 & 10 & 17 & 19 & 13 & 13 & 10 & 6 & 1 & 12 \\
24 & 2430 & 4 & 6 & 24 & 20 & 27 & 31 & 33 & 22 & 21 & 20 & 5 & 2 & 0 & 1
\end{tabular}

Während an normalen Kaninchen die renale Ausscheidung des peroral aufgenommenen Wassers überhaupt $z$ wischen $1 \frac{1}{2}-2 \frac{1}{2}$ Stunden ihren Höhepunkt erreicht, hat hier eine bedeutende Verzögerung derselben stattgefunden. 
IV. Wasserwechselstörungen bei Kaninchen mit Sonderfüterung von ungesättigten Fettsäuren.

In dieser Ver'suchsreihe wurden 4 ausgewachsene Kaninchen vorher neben der gewöhnlichen Laboratoriumnahrung auch täglich 3-5 $\mathrm{g}$ von Leinölsäure gegeben. Die Sonderfütterung erstreckte sich dann in einzelnen Tieren auf eine Zeitdauer von 1-2 Monaten fortwährend. Nach dieser Vorbehandlung wurde der eigentliche Wasserversuch auch in gleicher Weise ausgeführt, wie bei Kaninchen mit Toluylendiaminvergiftung. Wie das Resultat in der Tabelle 6 angeführt wird, hat man hier die halbstündliche Bestimmung des Hb-Gehaltes und der Harnmenge nach der peroralen Einführung von $150 \mathrm{ccm}$ physiologischer Kochsalzlösung in einer Sitzung vorgenommen.

\section{Tabelle 6.}

Versuche mit Leinölsäure.

\begin{tabular}{|c|c|c|c|c|c|c|c|c|c|c|c|c|c|}
\hline \multirow{2}{*}{$\begin{array}{l}\text { Nr.d. } \\
\text { Tiere }\end{array}$} & \multirow{2}{*}{$\begin{array}{c}\text { Ge- } \\
\text { wicht } \\
(g)\end{array}$} & \multirow{2}{*}{$\begin{array}{l}\text { Dauer } \\
\text { d. } \\
\text { Fütte- } \\
\text { rung }\end{array}$} & \multirow[b]{2}{*}{ Anfang } & \multicolumn{10}{|c|}{ Nach der Wasseraufnahme } \\
\hline & & & & St. $^{\frac{1}{2}}$ & $\begin{array}{c}1 \\
\text { St. }\end{array}$ & $\begin{array}{l}1 \frac{1}{2} \\
\text { St. }\end{array}$ & $\begin{array}{c}2 \\
\text { St. }\end{array}$ & $\begin{array}{l}2 \frac{1}{2} \\
\text { St. }\end{array}$ & $\begin{array}{c}3 \\
\text { St. }\end{array}$ & $\begin{array}{l}3 \frac{1}{2} \\
\text { St. }\end{array}$ & $\begin{array}{c}4 \\
\text { St. }\end{array}$ & $\begin{array}{l}4 \frac{1}{2} \\
\text { St. }\end{array}$ & $\begin{array}{c}\mathbf{5} \\
\text { St. }\end{array}$ \\
\hline 30 & 2800 & 59 Tage & $\begin{array}{l}\text { Hb. 15,42 } \\
\text { Ur. }\end{array}$ & $\left|\begin{array}{r}13,88 \\
0\end{array}\right|$ & $\begin{array}{r}13,42 \\
5\end{array}$ & & $\begin{array}{r}14,27 \\
15\end{array}$ & $\left|\begin{array}{r}15,15 \\
10\end{array}\right|$ & $\begin{array}{r}14,88 \\
0\end{array}$ & $\begin{array}{r}15,52 \\
10\end{array}$ & $\left|\begin{array}{r}14,82 \\
30\end{array}\right|$ & $\begin{array}{r}15,52 \\
10\end{array}$ & $\begin{array}{r}15,58 \\
0\end{array}$ \\
\hline 31 & 2050 & 56 Tage & $\begin{array}{l}\text { Hb. } 15,24 \\
\text { Ur. }\end{array}$ & $\begin{array}{r}13,71 \\
0\end{array} \mid$ & $\begin{array}{r}13,61 \\
0\end{array}$ & $\begin{array}{r}13,01 \\
10\end{array} \mid$ & $\begin{array}{r}14,32 \\
25\end{array}$ & 14,14 & $\begin{array}{r}14,35 \\
10\end{array}$ & $\begin{array}{r}14,88 \\
10\end{array}$ & $\begin{array}{r}14,35 \\
15\end{array}$ & & $\begin{array}{r}14,80 \\
0\end{array}$ \\
\hline 32 & 2410 & 32 Tage & $\begin{array}{l}\text { Hb. 14,94 } \\
\text { Ur. }\end{array}$ & & $\begin{array}{r}12,79 \\
25\end{array}$ & $\begin{array}{r}12,50 \\
20\end{array}$ & $\begin{array}{r}12,90 \\
15\end{array}$ & $\begin{array}{r}13,59 \\
10\end{array}$ & $\begin{array}{r}13,66 \\
20\end{array}$ & $\begin{array}{r}13,99 \\
15\end{array}$ & $\begin{array}{r}13,53 \\
5\end{array}$ & $\begin{array}{r}14,22 \\
5\end{array}$ & $\begin{array}{r}14,22 \\
0\end{array}$ \\
\hline 33 & 3240 & 32 Tage & $\begin{array}{l}\text { Hb. 14,52 } \\
\text { Ur. }\end{array}$ & $\begin{array}{r}13,39 \\
5\end{array}$ & $\begin{array}{r}12,37 \\
30\end{array}$ & $\begin{array}{r}12,64 \\
20\end{array}$ & $\begin{array}{r}13,03 \\
10\end{array}$ & $\begin{array}{r}13,44 \\
10\end{array}$ & $\begin{array}{r}13,73 \\
25\end{array}$ & $\begin{array}{r}14,60 \\
10\end{array}$ & $\begin{array}{r}14,85 \\
15\end{array}$ & $\begin{array}{r}14,49 \\
0\end{array}$ & $\begin{array}{r}14,43 \\
0\end{array}$ \\
\hline
\end{tabular}

Hb. ......Hb-Gehalt nach $B$ ü r ke r; Ur.......Harnmenge in ccm.

Die sekundäre Hydrämie trat hier fast gar nicht auf. Auch die renale Wasserausscheidung ist gewissermassen deutlich beeinträchtigt. Die Versuchsresultate decken sich also im wesentlichen mit denen der Toluylendiaminvergiftung.

\section{Störungen des intermediären Wasseraustausches bei Kaninchen mit gewisse Zeitlang fort- gesetzter Lezithinfütterung.}

In dieser Versuchsreihe standen zuerst 10 ausgewachsene Kaninchen zur Verfügung, deren Mehrzahl aber leider während der längere 
Zeit fortgesetzten Sonderfütterung verstorben war. Und nur bei drei Kaninchen konnte ich die angestellte Untersuchung in einem bestimmten Zeitabschnitt planmässig abschliessen. In der Tabelle 7 wird das Resultat bei einem Tier als ein Beispiel angeführt, bei dem der Wasserversuch in verschiedenen $Z$ wischenzeiten $4 \mathrm{mal}$ wiederholt durchgeführt wurde.

\section{Tabelle 7.}

Kaninchen Nr. 36, Gewicht 2735 g, während der ganzen Versuchszeit (von 7. September bis 27. Oktober) tägliche Fütterungsdosis des Lezithins von 1 bis auf $10 \mathrm{~g}$ allmählich gesteigert.

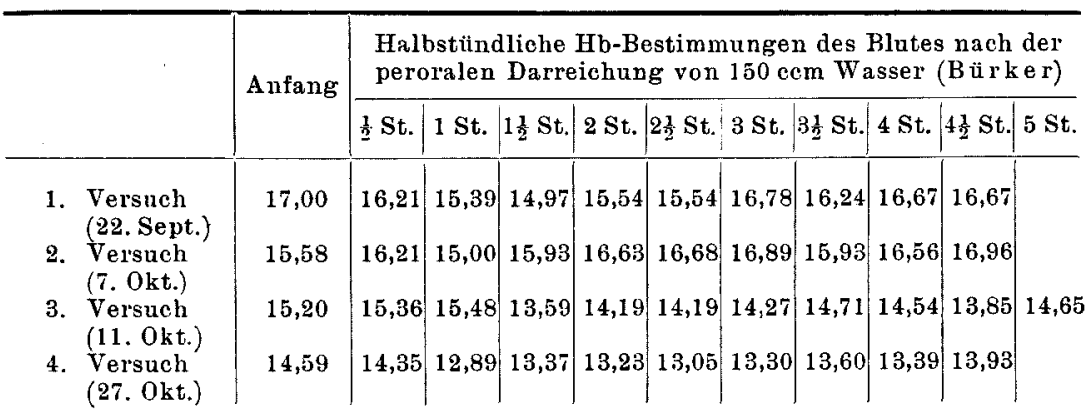

Schon der erste Wasserversuch ergab, dass die sekundäre Hydrämie hier nicht mehr als solche regelrecht auftrat. In späteren Stadien der fortgesetzten Lezithinfütterung liess sich eine bedeutende Beeinträchtigung des intermediären Wasseraustausches dadurch schliessen, dass neben dem mangelhaften Auftreten der sekundären Hydrämie auch die primäre nur in unbedeutender Weise zur Erscheinung trat, wie man in Fig. 3 leicht ersieht.

\section{Besprechung.}

Betrachtet man nun die ganzen Versuchsergebnisse hier zusammenfassend, so fällt zunächst auf, dass eine kleindosierte Toluylendiaminvergiftung beim Kaninchen ziemlich bedeutende Blutverände- 
rungen verursachte. Die Erythrozyten wiesen dabei zuerst eine geringe Vermehrung, dann aber doch fast ausnahmslos eine zunehmende Verminderung in der Weise auf, dass die betreffende Kurve zwischen der 3. und 6. Versuchswoche sehr steil abstieg und weiter dann mehr flach verlief. Dementsprechend hat auch der Hb-Gehalt allmählich abgenommen, wie die Kurve desselben ihr annähernd parallel ging. Demgegenüber haben die basophilen Erythrozyten in der 3 . Woche gewissermassen deutlich zugenommen. Sie begannen aber in der 4 . Woche wieder abzunehmen, dass von der 5 . Woche an ihre Zunahme iiberhaupt als nicht so bedeutend anzugeben war. Dabei handelte es sich wesentlich um eine Vermehrung von Polychromasie, die sich beim Kaninchen schon normalerweise in einer viel grösseren Menge befinden, als die basophile Punktierung. Die Leukozyten haben in der 1. Woche nur gering aber in der 2 . Woche etwas auffallender zugenommen, dass man hier im ganzen Verlauf von einer mässigen Leukozytose sprechen kann.

In gleicher Weise, wie die Toluylendiaminvergiftung entfaltete auch eine fortlaufende Fütterung mit Perilla-Öl oder Leinölsäure bei Kaninchen eine schädliche Wirkung, die hier ebenfalls eine hämolytische Anämie mit merklicher Vermehrung der basophilen Erythrozyten zur Folge hatte.

Wie ich bereits eingangs erwähnt, sind die Blutveränderungen bei Toluylendiaminvergiftung nach der Untersuchung von Joannovics und $\mathrm{Pick}^{4}$ im wesentlichen auf die hämolytische Wirkung der ungesättigten Fettsäuren zurückzuführen. Diese Annahme macht sich auch dadurch wahrscheinlich, dass meine Versuchsresultate mit Perilla-Öl oder Leinölsäure, die beide hauptsächlich bzw. ausschliesslich aus ungesättigten Fettsäuren bestehen, mit denen der Toluylendiaminvergiftung so gut wie vollkommen übereinstimmten.

Nach der Angabe von Koyanagi (1.c.) kommt bei der Retinitis nephritica recht häufig eine bedeutende Vermehrung der basophilen Erythrozyten im peripheren Blut vor, die hier ebenfalls als Ausdruck einer intravitalen Hämolyse aufzufassen ist. Diese Erscheinung erklärt sich dann durch eine einfache $\mathrm{H}_{y}$ percholesterinämie sehrschwer, ja fast unmöglich, wenn man einmal daran denkt, dass das Cholesterin eigentlich eine hemmende Wirkung auf hämolytische Einflüsse besitzt. Vielmehr muss in Betracht kommen eine Prävalenz der Phosphatide, die im Organismus hauptsächlich aus den ungesättigten Fett-

4) Joannovics u. Pick, Zeitschr. exp. Path. u. Th. 1907, 7, 185. 
säure als Antagonisten des Cholesterins bestehen. Die Blutveränderungen in meinen vorstehenden Versuchen sprechen nun gerade für die Annahme, dass bei Retinitis nephritica eine pathologischeZunahme der Phosphatide bzw. der Spaltungsprodukte derselben für ihre Entstehung möglicherweise eine bedeutende Rolle spielen wird.

In dem zweiten Abschnitt meiner Versuche habe ich mich mit der Frage beschäftigt, ob und wieweit eine übermässige Zufuhr der ungesättigten Fettsäuren auf den intermediären Wasseraustausch und auf die renale Ausscheidung schädliche Wirkungen ausübt. In einer Versuchsreihe dabei wurde eine subkutane Applikation des kleindosierten Toluylendiamins vorgenommen und in der anderen eine peropale Darreichung von Leinölsäure. Die beiden Versuchen ergaben in wesentlichen miteinander übereinstimmende Resultate, dass die ungesättigten Fettsäuren hier eine gestörte Durchlässigkeit der Kapillaren und gleichzeitig auch eine Funktionsstörung der Nieren hervorrufen können. Es ist weiter wohl nicht zu bezweifeln, dass die Wasserwechselstörungen, die in Folge der eine bestimmte Zeitlang fortgesetzten Lezithinfütterung auftraten, wesentlich auch auf einer übermässigen Aufnahme der ungesättigten Fettsäuren beruhen, die sich hier allerdings vom zugeführten Lezithin abspalteten. Diese Tatsache enthält nach meinem Dafürhalten für die Vermutung Koyanag is auch einen weiteren Beweis, dass zwischen dem erhöhten Gehalt an ungesättigten Fettsäuren des Blutes und den chronischen Nierenleiden mit Netzhautveränderungen eine kausale Beziehung bestünde, worauf auch anderweitige Versuchsresultate vou Rof u. Thurnherr ${ }^{5 j}$ bis zu einem gewissen Grade hinwiesen.

\section{Schluss.}

Eine fortlaufende übermässige Zufuhr von ungesättigten Fettsäuren im Organismus verursacht eine hämolytische Anämie mit merklicher Vermehrung von basophilen Erythrozyten im peripheren Blut sowie eine deutliche Störung des intermediären Wasseraustausches und der renalen Ausscheidung.

5) Rof u. Thurnherr, Zeitschr. exp. Med. 1933, 88, 693. 